\title{
Intestinal Apolipoprotein Synthesis in the Newborn Piglet
}

\author{
DENNIS D. BLACK AND PATRICIA L. ROHWER-NUTTER
}

Department of Pediatrics, University of Chicago, Pritzker School of Medicine, Chicago, Illinois 60637

\begin{abstract}
To determine the effects of dietary and biliary lipid absorption on intestinal apo B-48 and apo A-I synthesis in the newborn piglet, 2-d-old female piglets were prepared with a duodenal infusion catheter. After recovery, animals were given either low triglyceride (Vivonex; VIV group) or high triglyceride (Intralipid; FAT group) diets by continuous intraduodenal infusion for $24 \mathrm{~h}$. A bilediverted group was also studied. Segments of proximal jejunum and distal ileum were then pulse-radiolabeled in vivo with ${ }^{3} \mathrm{H}$-leucine. Mucosal apo B-48 and apo A-I were immunoprecipitated, and apoprotein synthesis was expressed as percentage of total protein synthesis. Mucosal apoprotein content (ng apoprotein/ $\mu \mathrm{g}$ total protein) was measured by competitive ELISA assays. In jejunum and ileum, apo B-48 synthesis was not different in the three groups. However, apo B content increased 2.4-fold in jejunum and 1.7-fold in ileum in the FAT group compared with the VIV group. Immunoblotting revealed the majority of jejunal apo $B$ to be apo B-48, not apo B-100 from contaminating plasma lipoproteins, in all three experimental groups. Bile-diverted animals had decreased jejunal apo B content compared with the VIV group. Jejunal apo A-I synthesis and content were approximately 2 -fold higher in FAT animals compared with the VIV group. Although ileal apo A-I synthesis was also 2-fold higher in the FAT group, apo A-I content was not different from the VIV group. Neither jejunal nor ileal apo A-I synthesis was significantly affected by bile diversion, even though jejunal apo A-I content was decreased by over two thirds compared with the VIV animals. In the newborn piglet, intestinal synthesis of apo B-48 and apo A-I is differentially regulated by luminal lipid absorption. Although fat feeding and bile diversion regulate mucosal apo B-48 content, synthesis is unchanged, indicating a posttranslational regulatory mechanism. In contrast, apo A-I synthesis generally parallels mucosal apo A-I content except in distal ileum. Changes in jejunal apo B content and apo A-I content and synthesis parallel changes in mucosal triglyceride content. However, changes in ileal apo B content and apo A-I synthesis were not accompanied by changes in ileal triglyceride content, suggesting other regulatory factors in the distal small intestine. (Pediatr Res 29: 32-38, 1991)
\end{abstract}

\section{Abbreviations}

VIV, receiving Vivonex

FAT, receiving Intralipid

BiD, bile-diverted
Apolipoproteins are the protein surface components of lipoprotein particles and serve essential functions in the secretion, metabolism, and receptor-mediated uptake of these particles. Apo $B$ is a component of triglyceride-rich lipoproteins and exists as two distinct forms in the human, rat, and swine (1-3). Apo $\mathrm{B}-100$ is the larger species and is a component of plasma VLDL and LDL and contains the LDL receptor binding domain (4). Apo B-48 is the smaller form and is found in intestinal chylomicrons and does not contain the LDL receptor binding sequence (5). In the postnatal human and swine, apo B-100 is of hepatic origin, and apo B-48 is synthesized by the intestine (1, $6)$. In both liver and intestine, apo $B$ is thought to be essential for the secretion of triglyceride-rich lipoproteins (3). Apo A-I is the major apolipoprotein of plasma HDL and is produced by both liver and intestine in the human, rat, and swine (1, 7-10). Its major metabolic role is that of an essential cofactor for lecithin: cholesterol acyltransferase, the enzyme responsible for the production of the majority of plasma cholesteryl esters (11).

Studies of the factors that regulate the intestinal synthesis of apolipoproteins in the developing mammal have been difficult to interpret because of the inability to separate the effect of dietary lipid absorption from that of factors such as changes in the hormonal milieu and other developmental cues in the various models used $(6,10,12-15)$. Rat pups are an altricial species (16), and dietary factors in the newborn are very difficult to manipulate in vivo. Ethical considerations preclude the extensive manipulation and tissue sampling of human subjects. Results of tissue culture studies from both the developing human and rat are difficult to interpret, particularly with regard to the effects of lipid absorption in vitro $(10,15)$. Furthermore, quantitation of specific apolipoprotein mRNA abundance alone will not detect potentially important co- and posttranslational regulatory mechanisms.

We have developed the neonatal piglet as an in vivo model for the study of the physiologic regulation of intestinal apolipoprotein synthesis by luminal lipid absorption in the immature mammal. Similarity with human physiology exists in many areas with regard to intestinal function and lipoprotein and apolipoprotein metabolism $(1,7,17,18)$. Thus far, the effects of dietary fat and biliary lipid absorption on the intestinal synthesis and secretion of apo B and apo A-I in the older suckling pig (1) and on intestinal apo A-IV gene expression in the fetal, newborn, and suckling animal (18) have been reported. Our study was undertaken to define the effects of dietary triglyceride and biliary lipid absorption on the intestinal synthesis of apo B-48 and apo A-I in the newborn piglet.

\section{MATERIALS AND METHODS}

Received February 16, 1990; accepted September 11, 1990.

Correspondence: Dennis D. Black, M.D. Department of Pediatrics, University of Chicago. Wyler Children's Hospital, 5825 South Maryland Avenue. Box 107 Chicago, IL 60637

Supported by NIH Grant HD-22551 and the Schweppe Foundation.
Animals. Two-d-old female domestic swine were obtained from Research Industries Corporation, Monee, IL.

Preparation of animal model. From the time of arrival to the time of surgery the next day, the 2-d-old animals were kept in 
heated isolettes. Animals were fed artificial sow's milk (SPFLAC, Pet-Ag, Inc., Hampshire, IL) by gavage during this period. Anesthesia was induced by intramuscular ketamine $(40 \mathrm{mg} / \mathrm{kg})$ and maintained by face mask delivering $1 \mathrm{~L} / \mathrm{min} \mathrm{O}_{2}$ and $0.5-$ $0.8 \%$ halothane. A longitudinal midline abdominal incision was made, and the peritoneal cavity opened. The second portion of the duodenum was cannulated with silicone rubber tubing (inner diameter 0.030 inch, outer diameter 0.065 inch) through an incision in the gastric antrum. The tubing was then secured in place with a purse-string suture, exteriorized through the right flank, tunneled s.c. to the mid-dorsum, and secured through a swivel tether. In bile-diverted animals, the common bile duct was cannulated with silicone rubber tubing (inner diameter 0.030 inch, outer diameter 0.065 inch) and was then doubly ligated distally. The cystic duct was also ligated, and the gallbladder was completely emptied by aspiration. The bile duct tubing was routed with the duodenal catheter through the flank and dorsum and into the tether. A dual channel tether was used with the bilediverted animals. Postoperatively, the animals were allowed to recover in the heated isolettes. An intraduodenal infusion of $5 \%$ glucose in $45 \mathrm{mM} \mathrm{NaCl}$ and $20 \mathrm{mM} \mathrm{KCl}$ at $100 \mathrm{~mL} / \mathrm{kg} / 24 \mathrm{~h}$ was started during recovery. In bile-diverted animals, extra fluid replacement was provided to compensate for bile drainage. After a 24 -h recovery period, animals were awake, alert, and mobile. Also at this time, animals were noted to be tolerating the infusion well without vomiting or abdominal distention and to be passing stool. Experimental infusions were started at the end of this recovery period.

After the 24-h recovery period, three groups of animals were studied, as illustrated in Figure 1: group 1, piglets receiving a 24 $h$ intraduodenal infusion of dilute Vivonex (Norwich Eaton Pharmaceuticals, Inc., Norwich, NY), a low-fat $(1.45 \mathrm{~g} / \mathrm{L}$ of fat in undiluted form) elemental formula, at $50 \mathrm{kcal} / \mathrm{kg} / 24 \mathrm{~h}$ (VIV group, $n=7$ ); group 2 , animals receiving a 24 -h intraduodenal infusion of Intralipid (Cutter, Berkeley, CA), a triglyceride emulsion, at $50 \mathrm{kcal} / \mathrm{kg} / 24 \mathrm{~h}$ (FAT group, $n=7$ ); and group 3, bilediverted animals receiving a $24-\mathrm{h}$ intraduodenal infusion of Vivonex at $50 \mathrm{kcal} / \mathrm{kg} / 24 \mathrm{~h}$ (BiD group, $n=4)$.

All groups received a total fluid volume of $100 \mathrm{~mL} / \mathrm{kg} / 24 \mathrm{~h}$

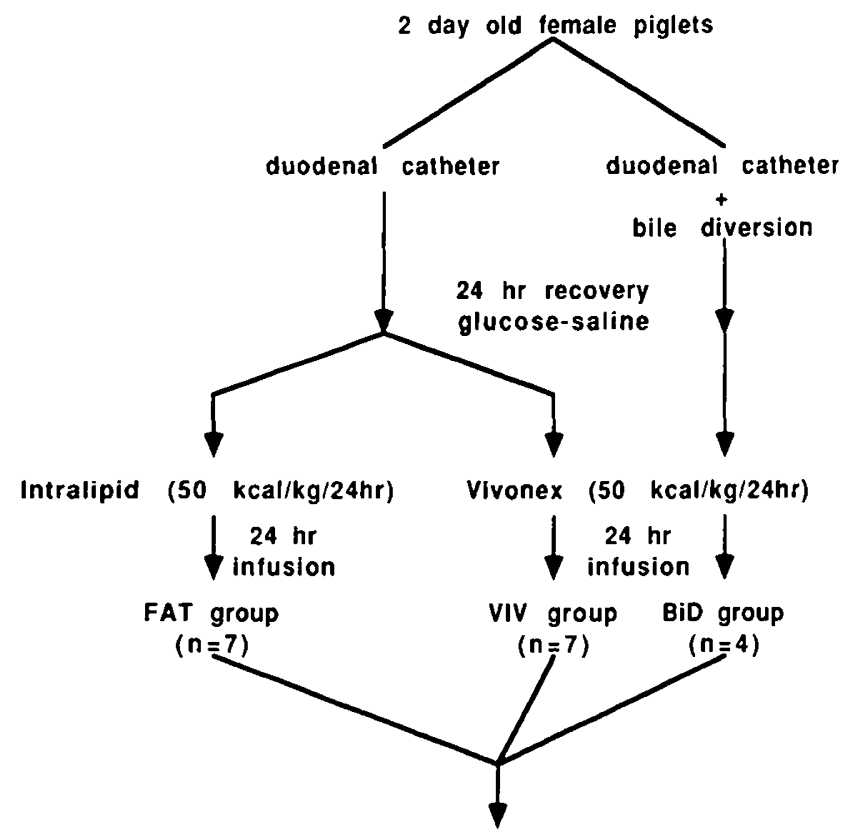

In vive pulse-radlolabeling of proximal jejunum and distal lleum with $3 \mathrm{H}$-leucine

Fig. 1. Experimental protocol for newborn pigiets. FAT animals reeived an intraduodenal infusion of Intralipid, VIV animals received an nfusion of Vivonex, and the bile-diverted (BiD) animals also received ne Vivonex infusion. during the experimental infusion using supplemental saline solution. The two experimental infusions were designed to differ as much as possible with regard to triglyceride content. A caloric intake of $50 \mathrm{kcal} / \mathrm{kg} / 24 \mathrm{~h}$ during the 24 -h experimental infusions was used to keep the infusions isocaloric and to take into account the fact that Vivonex has a high osmolality when used full strength $(550 \mathrm{mosmol} / \mathrm{kg})$, which was generally not tolerated by the animals. The $\mathrm{BiD}$ group also continued to receive bile drainage replacement in addition to the experimental infusion. During the infusions, the animals were kept in the heated isolettes and allowed to move freely within the limits of their tethers. At no time during the recovery phase or during the experimental infusions did the animals receive peroral feedings.

Determination of intestinal apolipoprotein synthesis. At the end of the experimental infusions, the animals were anesthetized, and a 10-cm segment of proximal jejunum was isolated $10 \mathrm{~cm}$ distal to the ligament of Treitz by two ligatures. Similarly, a 10$\mathrm{cm}$ segment of distal ileum was isolated $10 \mathrm{~cm}$ from the ileocecal valve. Radiolabeling was performed by instilling $1.5 \mathrm{mCi}$ of $\mathrm{L}-\left[4,5-{ }^{3} \mathrm{H}\right]$ leucine $(>120 \mathrm{Ci} / \mathrm{mmol}$ ) (Amersham, Arlington Heights, IL) into each segment. Nine min later, the segments were removed and prepared for immunoprecipitation as described below. This labeling time has previously been shown to be optimal in similar experiments in the adult rat, and we have used it previously in the piglet $(1,8,18,19)$. Also at this time, blood was collected by cardiac puncture into EDTA-containing tubes, and plasma was separated from red blood cells by centrifugation and stored with $0.01 \%$ EDTA, $0.02 \% \mathrm{Na}$ azide, $1 \mu \mathrm{g} /$ $\mathrm{mL}$ chloramphenicol, and $0.5 \mu \mathrm{g} / \mathrm{mL}$ gentamicin sulfate at $-80^{\circ} \mathrm{C}$ until apolipoprotein quantitation.

Preparation of mucosal cytosolic supernatants for immunoprecipitation. Radiolabeled intestinal segments were flushed with 50 $\mathrm{mL}$ of iced PBS (50 mM phosphate, $100 \mathrm{mM} \mathrm{NaCl}, \mathrm{pH} 7.4$ )- 20 $\mathrm{mM}$ leucine, and the mucosa was scraped and homogenized on ice in $1 \mathrm{~mL}$ of PBS-1\% Triton X-100-2 mM leucine- $1 \mathrm{mM}$

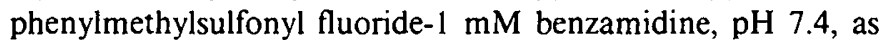
previously described $(1,18)$. Aliquots of the homogenate were taken for measurement of total protein concentration, trichloroacetic acid-precipitable radioactivity, and triglyceride content. The remainder was pelleted at $105000 \times g$ for $60 \mathrm{~min}$ in a 50.3 Ti rotor (Beckman Instruments, Palo Alto, CA), followed by collection of the cytosolic supernatant. Although most intracellular apolipoprotein is membrane-associated, this technique has been shown previously to result in extraction and solubilization of $84-94 \%$ of total recoverable apolipoprotein mass with no discernible effect of the state of lipid flux (19). All procedures were performed at $0-5^{\circ} \mathrm{C}$, and the mucosal supernatant samples were stored at $-80^{\circ} \mathrm{C}$ until analysis.

Apolipoprotein immunoprecipitation. Intestinal cytosolic supernatant fractions were subjected to specific immunoprecipitation of apo B and apo A-I under conditions of antibody excess as described $(1,18)$. Aliquots of cytosolic supernatants were mixed with washed IgG-Sorb (The Enzyme Center, Malden, MA) and subsequently reacted with excess antiapolipoprotein antiserum for $18 \mathrm{~h}$ at $4^{\circ} \mathrm{C}$. After a second addition of IgG-Sorb and extensive washing, the liberated immunocomplex was applied to either $5.6 \%$ (apo A-I) or 4\% (apo B) SDS polyacrylamide tube gels. After electrophoresis, gels were sliced into 1-mm slices and incubated in 3\% Protosol/97\% Econofluor (New England Nuclear, Boston, MA) at $37^{\circ} \mathrm{C}$ overnight before liquid scintillation counting in a Packard Model 2000 liquid scintillation counter (Packard Instruments, Downers Grove, IL). Apolipoprotein species were identified by comparison to stained coelectrophoresed apolipoproteins. Apolipoprotein synthesis rates were expressed as the percentage of specific immunoprecipitated apoprotein counts as compared with total protein trichloroacetic acid-precipitable counts. Apolipoprotein synthesis was thereby expressed as a percentage of total protein synthesis. All samples were subjected to reimmunoprecipitation to ensure the completeness of the first antigen-antibody reaction. 
APO B ELISA INHIBITION PLOT

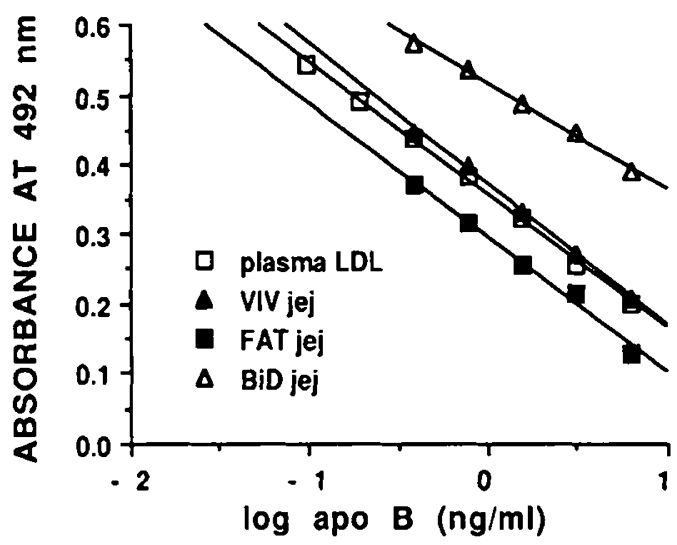

APO A-I ELISA INHIBITION PLOT

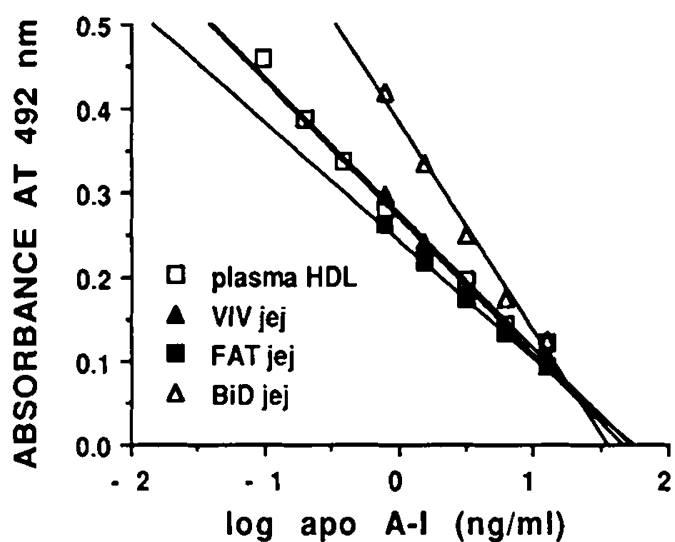

Fig. 2. Apolipoprotein ELISA semilogarithmic inhibition plots. Top panel (apo B assay): normal plasma LDL standard and jejunal cytosolic supernatants from VIV, FAT, and BiD animals. Bottom panel (apo A-I assay): normal plasma HDL standard and jejunal cytosolic supernatants from VIV, FAT, and BiD animals. The y axis indicates absorbance at $492 \mathrm{~nm}$. The $x$ axis indicates log apolipoprotein standard concentration ( $\mathrm{ng} / \mathrm{mL}$ ) in serial 1:2 dilutions. Supernatant samples were similarly applied to plates at 1:2 serial dilutions in parallel with the standards.

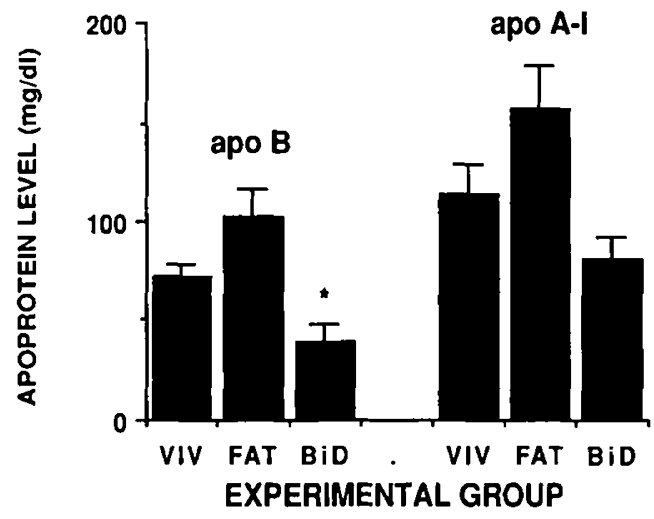

Fig. 3. Plasma apo B and apo A-I concentrations at the time of intestinal segment radiolabeling and removal as measured by ELISA. Bars represent mean \pm SEM. Significantly different from the VIV group at ${ }^{*} p<0.05$.

Apolipoprotein quantitation by ELISA. ELISA assays (20) were developed for swine apo B and apo A-I using polyclonal antibodies produced in New Zealand White rabbits after injection of electrophoretically pure pig apo B-100 from plasma LDL and apo A-I from plasma HDL. Briefly, 96-well microtiter plates

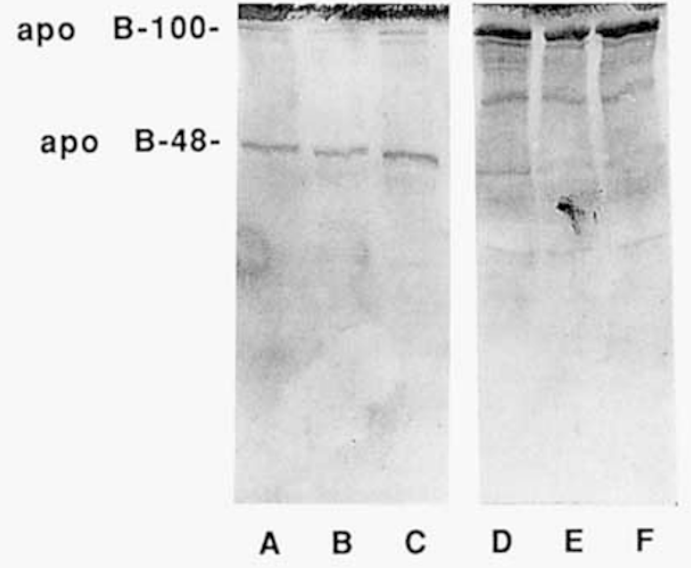

Fig. 4. Immunoblot of piglet jejunal cytosolic supernatants and whole plasma separated by $4 \%$ SDS-PAGE using rabbit anti-porcine $L D L$ apo B-100 antiserum as the primary antibody. Ten $\mu \mathrm{L}$ of either supernatant or plasma were applied to each lane. Lanes: $A$, VIV jejunum; $B$, FAT jejunum; $C$, BiD jejunum; $D$, VIV plasma; $E$, FAT plasma; and $F$, BiD plasma. Apo B-100 and B-48 were identified by comparison to coelectrophoresed prestained molecular weight standards. The plasma samples contain an intermediate molecular weight band that may be analogous to human apo B-74, a proteolytic product of apo B-100.

Table 1. Jejunal and ileal total protein sp act

\begin{tabular}{ccr}
\hline Experimental group & Jejunum & \multicolumn{1}{c}{ Ileum } \\
\hline VIV & $1138 \pm 212^{*}$ & $1097 \pm 275$ \\
FAT & $872 \pm 168$ & $867 \pm 144$ \\
BiD & $1147 \pm 306$ & $1136 \pm 265$ \\
\hline
\end{tabular}

* Expressed as $\mathrm{cpm} / \mu \mathrm{g}$ total protein in mucosal cytosolic supernatants (mean \pm SEM). No statistically significant difference was found among any of the values.

(Falcon, Becton Dickinson and Co., Oxnard, CA) were coated with either purified swine plasma LDL (apo B assay) or HDL (apo A-I assay) at $100 \mathrm{ng}$ protein/well. Competition assays were performed using serial dilutions of either plasma samples or cytosolic supernatants prepared from the tissue homogenates as the competing antigen. After incubation with biotinylated goat anti-rabbit IgG (Vector Laboratories, Burlingame, CA) followed by avidin-biotinylated peroxidase complex (Vectastain, Vector Laboratories), color was developed by addition of 0 -phenylenediamine (20). Color development was stopped by the addition of $2 \mathrm{M}$ sulfuric acid. Standard antigens consisted of swine plasma LDL (apo B) and HDL (apo A-I) applied to the microtiter plate wells in serial dilutions. Assay plates were read at $492 \mathrm{~nm}$ by a Bio-Rad ELISA reader (Bio-Rad Laboratories, Richmond, CA) interfaced with an Apple Macintosh plus microcomputer (Apple Computer, Inc., Cupertino, CA) and associated Bio-Rad software. Figure 2 shows ELISA semilogarithmic inhibition plots for plasma apolipoprotein standards and intestinal cytosolic supernatants from each of the three experimental groups of animals for apo B (top panel) and apo A-I (bottom panel). All plots are parallel to the standard plots, except for the BiD samples, whict: have slightly different slopes compared with the standards. How. ever, we judged the slopes to be close enough to allow relative quantitation in the BiD animals. All samples were run in dupli. cate in the same assay, and variability between duplicates wa: less than $5 \%$.

Immunoblotting. Aliquots of whole plasma and intestinal mu cosa cytosolic supernatants were subjected to $4 \%$ SDS-PAGF under reducing conditions according to the method of Laemml (21). Prestained molecular weight standards (BRL, Life Tech nologies, Inc., Gaithersburg, MD) were coelectrophoresed witl the samples. Electrophoresed proteins were then transferred to nitrocellulose paper and immunostained with swine polyclona 

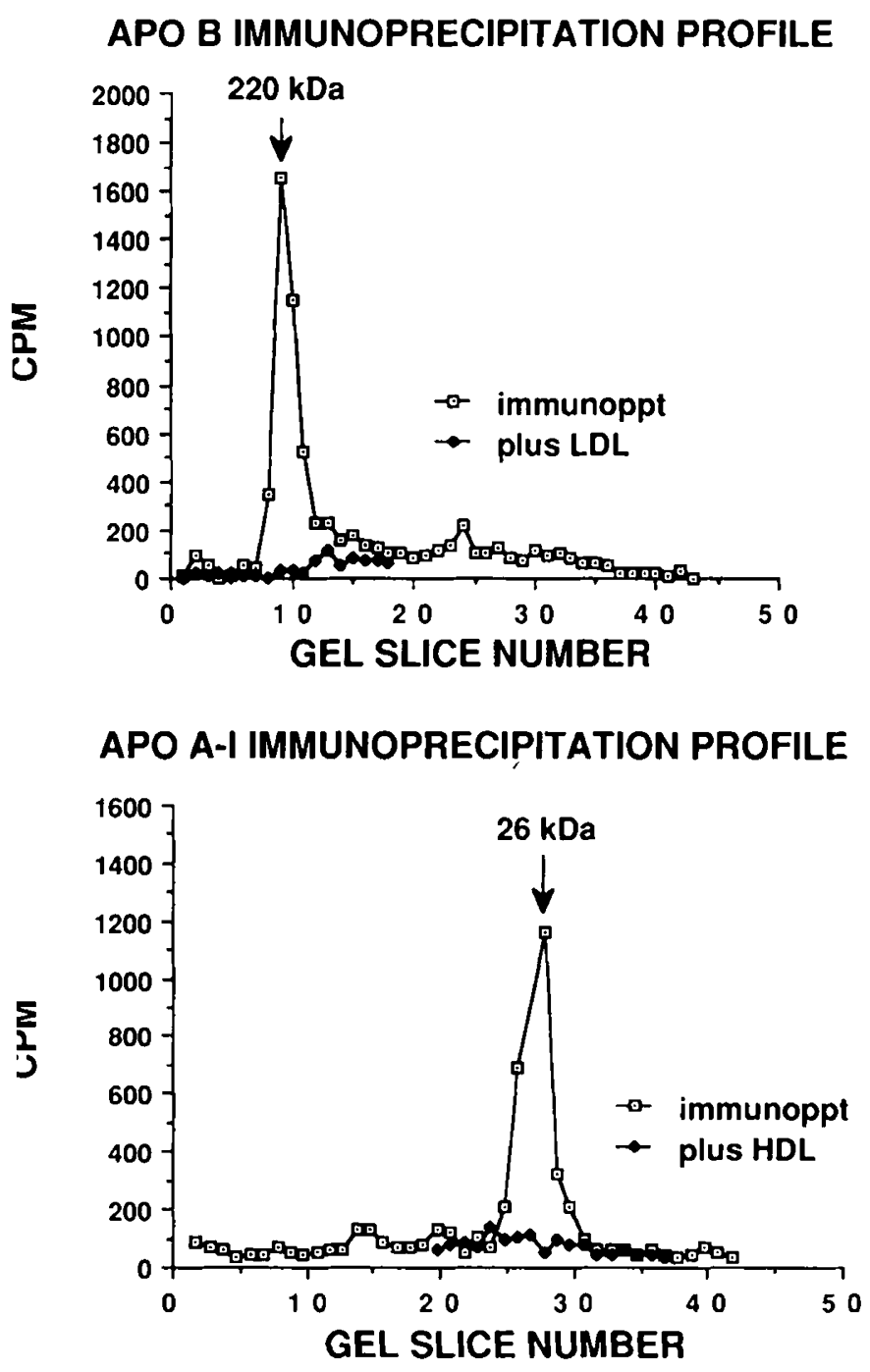

Fig. 5. Electrophoretic profiles of apo B-48 (top panel) and apo A-I (bottom panel) radiolabeled immunoprecipitates separated by $4 \%$ (apo B-48) and 5.6\% (apo A-I) SDS-PAGE followed by gel slicing and scintillation counting. Molecular weights were determined by comparison to stained coelectrophoresed molecular weight standards. As shown, immunoprecipitation of apo B-48 and apo A-I is specifically blocked by incubation with swine LDL and $\mathrm{HDL}$, respectively.

apo B antiserum as described (22) using 1:100 and 1:3000 dilutions of primary and secondary antisera, respectively.

Protein and triglyceride measurement. Protein was measured by a modified Lowry technique (23). Total lipid was extracted from aliquots of the mucosal homogenates by the method of Folch (24), followed by triglyceride measurement using an enzymatic assay (Sigma Chemical Co., St. Louis, MO).

Statistical analysis. Data in experimental groups were analyzed by one-way analysis of variance followed by the Student-Newman-Keuls test to compare specific groups. The null hypothesis was rejected at $p<0.05$.

\section{RESULTS}

Plasma apolipoprotein concentrations. Plasma apo B and apo 4-I concentrations of animals at the time they were killed are shown in Figure 3. Mean apolipoprotein levels tended to be aigher in FAT animals and lower in BiD animals as compared with the VIV group. However, statistical significance was achieved only for apo $\mathrm{B}$ levels in the $\mathrm{BiD}$ group. To determine he major apo B species (apo B-48 of intestinal origin versus apo 3-100 of hepatic origin) contributing to the total plasma apo B
JEJUNAL APOLIPOPROTEIN SYNTHESIS

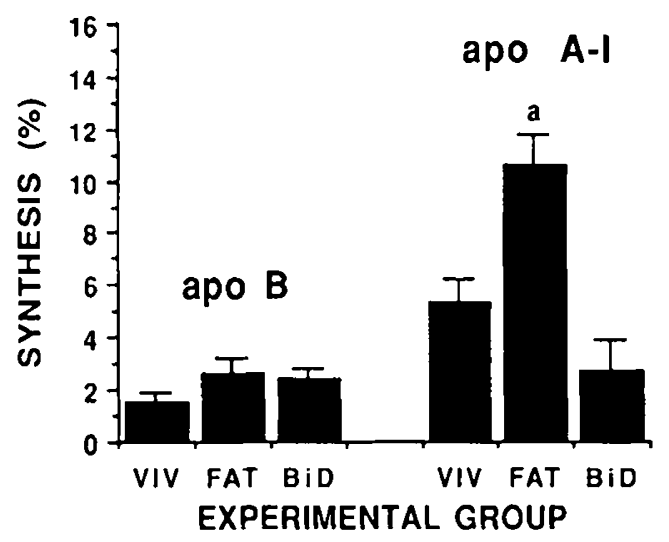

JEJUNAL APOLIPOPROTEIN CONTENT

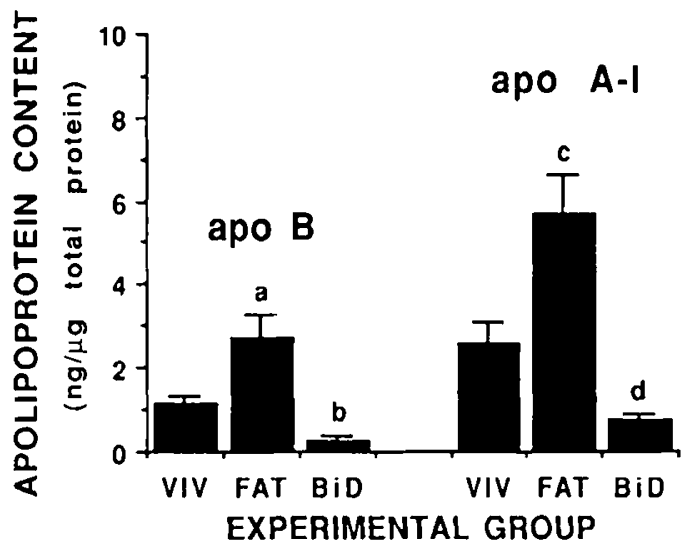

Fig. 6. Newborn piglet jejunal apo $B$ and apo A-I synthesis (top panet) and mucosal content (bottom panel) in the three experimental groups of animals. Apoprotein synthesis is expressed as percentage of total protein synthesis as determined by specific immunoprecipitated apoprotein cpm/ trichloroacetic acid precipitable $\mathrm{cpm} \times 100$. Mucosal apoprotein content was measured by ELISA and expressed as ng apoprotein/ $\mu \mathrm{g}$ total protein. Bars represent mean \pm SEM. Top panet: significantly different from the VIV group at ${ }^{a} p<0.01$. Bottom panel: significantly different from the VIV group at ${ }^{\circ} p<0.01,{ }^{b} p<0.05,{ }^{\circ} p=0.01$, and ${ }^{\mathrm{d}} p<0.05$.

Table 2. Mucosal triglyceride content

\begin{tabular}{ccc}
\hline Experimental group & Jejunum & Ileum \\
\hline VIV & $14.6 \pm 1.9^{*}$ & $9.8 \pm 2.1$ \\
FAT & $141 \pm 63 \dagger$ & $10.8 \pm 2.0 \ddagger$ \\
BiD & $5.0 \pm 1.2 \dagger$ & $12.1 \pm 4.0$ \\
\hline
\end{tabular}

* Mucosal homogenate triglyceride content expressed as $\mu \mathrm{g} / \mathrm{mg}$ total protein (mean \pm SEM).

$\dagger$ Significantly different from the VIV jejunal value at $p<0.05$.

$\ddagger$ Significantly different from the FAT jejunal value at $p<0.05$.

level, an aliquot of whole plasma from an animal in each experimental group was subjected to SDS-PAGE followed by electrotransfer to nitrocellulose and immunoblotting with rabbit anti-swine apo $B$ polyclonal antibodies reactive to both apo $B$ species (Fig. 4). As shown, apo B-100 is the predominant plasma species in all animal groups with only a small amount of apo B48 present. An intermediate molecular weight band is also seen that may be analogous to human apo B-74, a proteolytic product of apo B-100 $(2,3)$. Therefore, under all conditions of intestinal lipid flux, the major source of plasma apo $B$ appeared to be the liver.

Intestinal apolipoprotein synthesis and content. Incorporation of ${ }^{3} \mathrm{H}$-leucine into mucosal total protein in both jejunal and ileal 


\section{ILEAL APOLIPOPROTEIN SYNTHESIS}

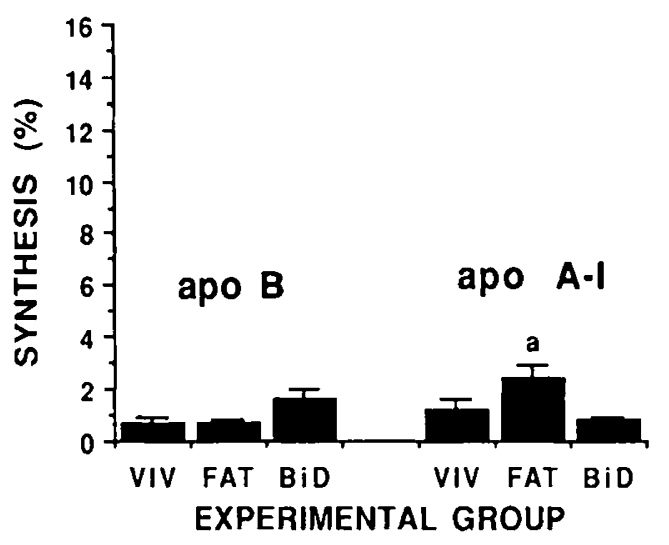

\section{ILEAL APOLIPOPROTEIN CONTENT}

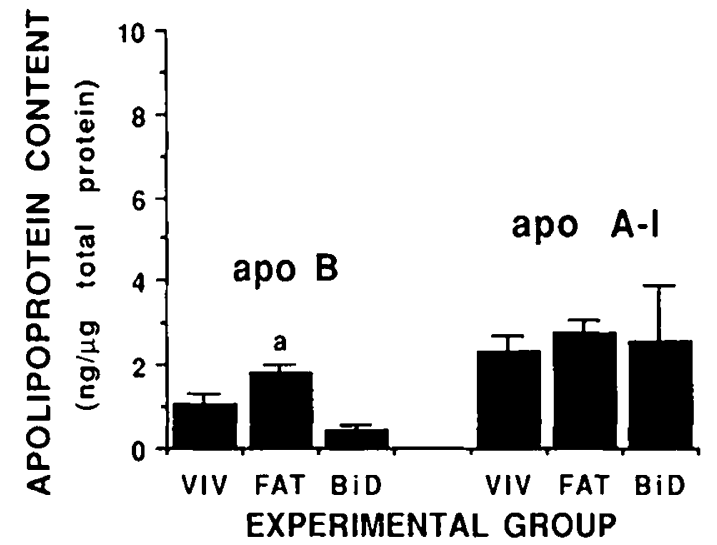

Fig. 7. Newborn pigiet ileal apo B and apo A-I synthesis (top panel) and mucosal content (bottom panel) in the three experimental groups of animals. Apoprotein synthesis and content were determined and expressed as described for Figure 6. Bars represent mean \pm SEM. Top panel: significantly different from the VIV group at ${ }^{a} p<0.05$. Bottom panel: significantly different from the VIV group at ${ }^{\circ} p<0.05$.

segments in all three experimental groups was comparable, as shown in Table 1. Figure 5, top panel, shows a typical $4 \%$ SDSPAGE gel profile of the apo $\mathrm{B}$ immunocomplex isolated from radiolabeled proximal jejunal mucosa of a FAT animal. Only apo B-48 (apparent mol wt approximately 220000 D) synthesis is present; no apo B-100 (apparent mol wt approximately $330000 \mathrm{D})$ peak is seen. In addition, competition with excess pig plasma LDL completely blocked the apo B-48 immunoprecipitation. Only apo B-48, not apo B-100, synthesis was found in all experimental groups in both proximal and distal small intestine.

Figure 5, bottom panel, shows a typical 5.6\% SDS-PAGE gel profile of the apo A-I (apparent mol wt approximately 26000 D) immunocomplex isolated from the same animal as for apo B-48 above. Competition with excess pig plasma HDL completely blocked the immunoprecipitation.

Jejunal apo B-48 and apo A-I synthesis rates for the three experimental groups are shown in Figure 6, top panel. Neither absorption of dietary triglyceride nor removal of biliary lipid had any effect on apo B-48 synthesis. However, jejunal mucosal apo B content (Fig. 6, bottom panel) more than doubled with triglyceride absorption and decreased by three fourths with bile diversion, as compared with the VIV group. Immunoblotting (Fig. 4) demonstrates that in FAT, VIV, and BiD jejunal homogenates, apo $B$ is present almost exclusively as apo B-48, thus ruling out mucosal contamination with plasma apo B-100 as the source of the observed changes in apo B content.
Jejunal apo A-I synthesis approximately doubled with triglyceride absorption (Fig. 6, top panel). The rate in the bile-diverted animals was decreased compared with the VIV group, but did not achieve statistical significance. Jejunal apo A-I content significantly increased with fat feeding and decreased with bile diversion (Fig. 6, bottom panel). Therefore, in contrast to apo B48 , jejunal apo A-I synthesis is regulated by dietary triglyceride absorption and parallels mucosal content. Table 2 shows a 10 fold increase in jejunal mucosal triglyceride content in the FAT group compared with the VIV group. In the BiD group, jejunal triglyceride content was depleted by two thirds as compared with the VIV group. Therefore, in jejunum the changes in apo B content and in apo A-I synthesis and content appear to be correlated with changes in mucosal triglyceride content.

Ileal apo B-48 synthesis did not change significantly with either fat feeding or bile diversion (Fig. 7, top panel), as noted in the jejunum. However, ileal apo B content increased significantly with triglyceride absorption (Fig. 7, bottom panel). Bile diversion decreased ileal apo B content, but not to a statistically significant level. Ileal apo A-I synthesis doubled with fat feeding, but was not altered significantly by bile diversion (Fig. 7, top panel). Ileal apo A-I content did not change with either triglyceride absorption or diversion of biliary lipid (Fig. 7, bottom panel). In contrast to findings in the jejunum, ileal triglyceride content was not significantly different in the three groups (Table 2), despite the observed changes in apo B content and apo A-I synthesis.

\section{DISCUSSION}

Our studies were undertaken to determine the regulatory effects of dietary triglyceride and biliary lipid absorption on intestinal synthesis of apo B and apo A-I in the newborn piglet, a model for the neonatal mammal. This model offers several advantages, including homology with human apolipoproteins, easy surgical manipulation to allow controlled delivery of experimental diets directly into the proximal small intestine and biliary diversion, and the capability of determination of apolipoprotein synthesis in vivo $(1,18)$.

Our studies demonstrate that the newborn piglet small intestine synthesizes only apo B-48, as determined by the size of the apo B immunoprecipitate in SDS-PAGE gels. Glickman, Rogers, and Glickman (6) have demonstrated the intestinal synthesis of apo B-100 in the human fetus with a gradual switch to apo B-48 during gestation. No data is available from the human newborn. Recent studies in the adult rabbit, human, and rat have shown that apo B-100 and apo B-48 are translated from the same mRNA sequence with the tissue-specific posttranscriptional insertion of a stop codon limiting translation to apo B-48 synthesis in the intestine $(25,26)$. If the same mechanism is operative in the newborn piglet intestine, it appears that the translatable apo B mRNA consists exclusively of the form encoding apo B-48.

Despite an increase in mucosal apo B content with triglyceride absorption in both jejunum and ileum in the newborn swine, the synthesis of apo B-48 did not significantly change. Immunoblotting revealed the majority of the jejunal mucosal apo $B$ to be apo B-48, making the possibility that the change was due to contamination by plasma apo B-100 very unlikely. This finding is similar to that noted previously in the older suckling swine where an increase in mesenteric lymph output of apo B with triglyceride absorption was not accompanied by increased mucosal synthesis (1). A similar observation has also been made in the adult rat (19). Inasmuch as total mucosal apo B, including intracellular and newly secreted extracellular apolipoprotein, was quantitated in our studies, changes in the intracellular pool of apo B cannot be determined. Studies in cultured rat hepatocytes suggest that significant amounts of newly synthesized apo $B$ are degraded intracellularly (27). Furthermore, oleate added to the medium of cultured HepG2 cells has been shown to stimulate apo B secretion without an accompanying increase in apo B mRNA abundance (28). It is an attractive hypothesis that in the 
absence of luminal lipid absorption newly synthesized intestinal apo B is rapidly degraded intracellularly until it is needed for lipoprotein packaging and secretion. This would account for the observed changes in mucosal apo B mass with triglyceride absorption and biliary diversion without a concomitant change in synthesis. The further study of this phenomenon on the cellular level in the piglet small intestine is underway in our laboratory.

As noted previously in the older suckling piglet (1), biliary diversion did not change apo B-48 synthesis in either jejunum or ileum of the newborn animal, although mucosal mass was reduced significantly in jejunal mucosa. Previous studies in the adult rat have suggested a major contribution of biliary lipid to the basal expression of intestinal apo B synthesis (29). However, at early developmental stages in the piglet, apo B synthesis appears to be fixed at a constant rate and is not acutely regulated by either dietary or biliary lipid absorption.

In contrast to the absence of apo B synthetic regulation by triglyceride absorption in the newborn piglet, apo A-I synthesis doubles in both jejunum and ileum with an accompanying increase in mucosal apo A-I mass in jejunum only. Interestingly, this responsiveness of intestinal apo A-I synthesis to lipid absorption is not present in the older suckling pig (1), and is also not present in the adult rat (8). Therefore, the loss of this synthetic regulation outside of the early neonatal period appears to be developmentally determined. However, the exact mechanisms, such as developmental changes in bowel surface area, absorptive efficiency, the enterocyte hormonal milieu, and preprogrammed developmental cues, remain to be elucidated. Removal of biliary lipid absorption did not significantly affect intestinal apo A-I synthesis in either jejunum or ileum, although mucosal apo A-I content was significantly reduced in the jejunum. This lack of apo A-I synthetic regulation by biliary lipid was also noted previously in older suckling swine (1), although bile diversion in the adult rat does reduce ileal apo A-I synthesis (8). This difference may be species-specific.

The mechanism by which changes in mucosal lipid flux might mediate the observed changes in apolipoprotein synthesis and content cannot be fully inferred from our results. In jejunum, apo B content and apo A-I synthesis and content tended to parallel changes in mucosal triglyceride content, as triglyceride absorption increased mucosal triglyceride content 10 -fold and biliary diversion caused depletion by two thirds. This suggests that fat absorption may influence apolipoprotein synthesis and content. However, one cannot infer flux or absolute absorption rates from measurement of triglyceride content. In contrast to findings in the jejunum, ileal triglyceride content was unaffected by triglyceride infusion or biliary diversion. Therefore, the observed changes in ileal apo B content and apo A-I synthesis may be mediated by mechanisms other than triglyceride absorption, but to unequivocally prove this would require measurement of triglyceride flux across the regional mucosa. Other important regulatory factors may include absorption of biliary phospholipid or bile salts, which would be increased during fat feeding and eliminated during biliary diversion. It is also conceivable that gut-derived hormones released with feeding, such as gastrin, may have an effect on apolipoprotein metabolism in the distal small intestine.

Analysis of plasma apolipoprotein concentrations in our study generally demonstrated an increase in apo B and apo A-I levels with lipid absorption and a reduction in levels with biliary diversion. However, statistical significance was achieved only for apo $B$ in the bile-diverted animals. Because immunoblotting revealed the majority of plasma apo B to be apo B-100 in all groups and piglet liver has been previously shown to synthesize exclusively apo B-100 (1), we speculate that biliary diversion may regulate plasma apo B-100 levels by changes in hepatic secretion, as well as by changes in plasma apolipoprotein clearance rates. Delineation of the exact mechanism will require further study. The low proportion of apo B-48 in the plasma of FAT animals by immunoblotting suggests that intestinally de- rived apo B-48-containing lipoproteins, including chylomicrons, are rapidly cleared from the plasma compartment.

Intestinal synthesis of apo B-48 and that of apo A-I appears to be differentially regulated by lipid absorption in the newborn piglet. Most striking is the apparent posttranslational regulation of apo B-48 mucosal content with luminal lipid absorption, as compared with the parallel changes in jejunal synthesis and content noted for apo A-I. In other studies, we have demonstrated a 7-fold up-regulation of proximal intestinal apo A-IV synthesis and mRNA abundance in the newborn piglet with triglyceride absorption with biliary lipid absorption having no measurable effect (18). Future studies in our laboratory will focus on the cellular mechanisms of these regulatory patterns and their integration with the packaging and secretion of nascent lipoproteins in the small intestine of the developing mammal.

Acknowledgments. The authors thank Dr. Bernard Davis for his helpful advice in the development of the apolipoprotein ELISA assays. Helpful suggestions by Dr. Nicholas Davidson were appreciated.

\section{REFERENCES}

1. Black DD, Davidson NO 1989 Intestinal apolipoprotein synthesis and secretion in the suckling pig. J Lipid Res 30:207-218

2. Kane JP 1983 Apolipoprotein B: structural and metabolic heterogeneity. Ann Rev Physiol 45:637-650

3. Olofsson S-O, Bjursell G, Bostrom K, Carlsson P, Elovson J, Protter AA Reuben MA. Bondjers G 1987 Apolipoprotein B: structure, biosynthesis, and role in the lipoprotein assembly process. Atherosclerosis $68: 1-17$

4. Knott TJ, Pease RJ, Powell LM, Wallis SC, Rall SC, Innerarity TL, Blackhan B, Taylor WH, Marcel Y, Milne R, Johnson D, Fuller M, Lusis AJ, McCarthy BJ, Mahley RW, Levy-Wilson B, Scott J 1986 Complete protein sequence and identification of structural domains of human apolipoprotein B-100. Nature 323:734-738

5. Hui DY, Innerarity TL, Milne RW, Marcel YL, Mahley RW 1984 Binding of chylomicron remnants and beta VLDL to hepatic and extra-hepatic lipoprotein receptors: a process independent of apolipoprotein B-48. J Biol Chem 259:15060-15068

6. Glickman RM, Rogers M, Glickman JN 1986 Apolipoprotein B synthesis by human liver and intestine in vitro. Proc Natl Acad Sci USA 83:5296-5300

7. Chapman MJ 1986 Comparative analysis of mammalian plasma lipoproteins. Methods Enzymol 128:70-143

8. Davidson NO, Glickman RM 1985 Apolipoprotein A-I synthesis in rat small intestine: regulation by dietary triglyceride and biliary lipid. J Lipid Res 26:368-379

9. Wu AL, Windmueller HG 1979 Relative contribution by liver and intestine to individual plasma apolipoproteins in the rat. J Biol Chem 254:7316-7322

10. Zannis VI, Kurnit DM, Breslow JL 1982 Hepatic apo A-I and apo E and intestinal apo A-I are synthesized in precursor isoprotein forms by organ cultures of human fetal tissues. J Biol Chem 257:536-544

11. Fielding CJ, Shore VG, Fielding PE 1972 A protein co-factor of lecithin: cholesterol acyltransferase. Biochem Biophys Res Commun 46:1493-1498

12. Demmer LA, Levin MS, Elovson J, Reuben MA, Lusis AJ, Gordon JI 1986 Tissue-specific expression and developmental regulation of the rat apolipoprotein B gene. Proc Natl Acad Sci USA 83:8102-8106

13. Elshourbagy NA, Boguski MS, Liao WSL, Jefferson LS, Gordon JI, Taylor JM 1985 Expression of rat apolipoprotein A-IV and A-I genes: mRNA induction during development and in response to glucocorticoids and insulin. Proc Natl Acad Sci USA 82:8242-8246

14. Fernando-Warnakulasuriya GJP, Eckerson ML, Clark WA. Wells MA 1983 Lipoprotein metabolism in the suckling rat: characterization of plasma and lymphatic lipoproteins. J Lipid Res 24:1626-1638

15. Perrin-Ansart MC, Vacher D, Girard-Globa A 1988 Determination of apolipoprotein A-I synthesis in intestinal explants from fetal and neonatal rats. Biochim Biophys Acta 963:541-548

16. Henning SJ 1981 Postnatal development: coordination of feeding, digestion, and metabolism. Am J Physiol 241:G199-G214

17. Corring $T$, Durand G, Henry Y 1982 Some aspects of development and nutrition in the monogastric animal during postnatal life. World Rev Nutr Diet 39:124-190

18. Black DD, Rohwer-Nutter PL, Davidson NO 1990 Intestinal apolipoprotein A-IV gene expression in the piglet. J Lipid Res 31:497-505

19. Davidson NO, Kollmer ME, Glickman RM 1986 Apolipoprotein B synthesis in rat small intestine: regulation by dietary triglyceride and biliary lipid. $\mathrm{J}$ Lipid Res 27:30-39

20. Madri JA, Barwick KW 1983 Methods in laboratory investigation: use of avidin biotin complex in an ELISA system: a quantitative comparison with two other immunoperoxidase detection systems using keratin antisera. Lab Invest 48:98-107

21. Laemmli UK 1970 Cleavage of structural proteins during assembly of the head of bacteriophage T4. Nature 227:680-685 
22. Towbin H, Staehelin T, Gordon J 1979 Electrophoretic transfer of proteins from polyacrylamide gels to nitrocellulose sheets: procedure and some applications. Proc Natl Acad Sci USA 76:4350-4354

23. Markwell MK, Haas SM, Bieber LL, Tolbert NE 1978 A modification of the Lowry procedure to simplify protein determination in membrane and lipoprotein samples. Anal Biochem 87:206-210

24. Folch J, Lees M, Sloane Stanley GH 1957 A simple method for the isolation and purification of tolal lipids from animal tissues. J Biol Chem 226:497509

25. Chen S-H, Habib G, Yang C-Y, Gu Z-W, Lee BR, Weng S-A, Silberman SR Cai S-J, Deslypere JP, Rosseneu M, Gotto AM, Lee W-H, Chan L 1987 Apolipoprotein B-48 is the product of a messenger RNA with an organspecific in-frame stop codon. Science 238:363-366
26. Powell LM. Wallis SC, Pease RJ, Edwards YH, Knott TJ, Scott J 1987 A novel form of tissue-specific RNA processing produces apolipoprotein B-48 in intestine. Cell 50:831-840

27. Borchardt RA, Davis RA 1987 Intrahepatic assembly of very low density lipoproteins. J Biol Chem 262:16394-16402

28. Pullinger CR, North JD, Teng B-B, Rifici VA, Ronhild de Brito AE, Scott J 1989 The apolipoprotein B gene is constitutively expressed in HepG2 cells: regulation of secretion by oleic acid, albumin, and insulin, and measurement of the mRNA half-life. J Lipid Res 30:1065-1077

29. Davidson NO, Drewek MJ, Gordon JI, Elovson J 1988 Rat intestinal apolipoprotein $\mathrm{B}$ gene expression: evidence for integrated regulation by bile salt fatty acid, and phospholipid flux. J Clin Invest 82:300-308 